\title{
Morphophysiology and Cryopreservation of Seeds of Dendrobium Nobile Lindl. (Orchidaceae) at Different Stages of Development
}

\section{Galina L Kolomeitseva}

N.V. Tsitsin Main Botanical Garden of Russian Academy of Sciences

\section{Tatiana V Nikishina}

K.A. Timiryazev Institute of Plant Physiology of Russian Academy of Sciences

\section{Alexander V Babosha}

N.V. Tsitsin Main Botanical Garden of Russian Academy of Sciences

Andrey S Ryabchenko ( $\square$ marchellos@yandex.ru )

N.V. Tsitsin Main Botanical Garden of Russian Academy of Sciences https://orcid.org/0000-00015200-1273

Olga N Vysotskaya

K.A. Timiryazev Institute of Plant Physiology of Russian Academy of Sciences

\section{Research Article}

Keywords: Orchids, Cryopreservation, In vitro, Immature seeds, Embryogenesis, Vital dyes

Posted Date: June 3rd, 2021

DOl: https://doi.org/10.21203/rs.3.rs-471835/v1

License: (c) (1) This work is licensed under a Creative Commons Attribution 4.0 International License.

Read Full License

Version of Record: A version of this preprint was published at Acta Physiologiae Plantarum on February 5th, 2022. See the published version at https://doi.org/10.1007/s11738-022-03372-z. 


\section{Abstract}

We studied the seed maturation of Dendrobium nobile Lindl to establish the optimal stage for green pod culture and long-term storage in liquid nitrogen. It was found that the immature seeds can germinate in vitro starting from 3 months after pollination (MAP) but only develop up to the stage of embryo swelling or protocorm without rhizoids. The maximum staining of embryos with vital dyes FDA and TTC occurs at 4 and 5 MAP that corresponds to the release of suspensor beyond the embryo sac. Embryo staining does not correlate with germination capacity and seed viability in cryogenic storage. The immature seeds acquire resistance to freezing and drying in airflow at the age of 6 MAP. The time of technical maturity for green pod culture and cryopreservation may be reduced from 12 MAP to 6-7 MAP.

\section{Key Message}

The immature seeds of Dendrobium nobile acquire resistance to freezing and long-term storage in a cryobank at the age of 6 MAP.

\section{Main Text}

The ongoing interest in orchids cultivation is explained not only by the ornamental features of the flowers but also by diverse biologically active compounds, produced in their stems and leaves. Dendrobium nobile Lindl. are widely used in traditional eastern and modern western medicine.

One of the ways to optimize the in vitro orchid cultivation may be a green pod culture in which immature seeds are germinated (Vasudevan and van Staden 2010; Udomdee et al. 2014). Collection orchids cultivated in the greenhouse are severely depleted during fruiting, therefore, it is desirable to reduce the maturation period.

However, the requirements for seed germination in vitro and cryogenic storage are different. Immature seeds that contain a lot of water in cells are better for in vitro germination, and mature dried seeds are better for cryogenic storage.

The work aims to determine the time of morphophysiological maturity of Dendrobium nobile seeds for the green pod culture and long-term storage in liquid nitrogen.

Plants of Dendrobium nobile Lindl. (Orchidaceae Juss.) (Fig. 1A) were cultivated in the greenhouse with a moderately warm temperature regime (day $18-26^{\circ} \mathrm{C}$, night $14-18{ }^{\circ} \mathrm{C}$ ) at a relative humidity of $60-75 \%$ and natural light. The flowering of different clones continued from November to March. The fruits were obtained by artificial allogamous pollination. The age of the seeds and ovules was calculated in months after pollination (MAP).

Germination ability was evaluated before and after cryogenic storage of seeds. The fruits were surface sterilized with $70 \%$ ethanol. The seeds were extracted with a sterile tool. More mature seeds (7-12 MAP) 
were sterilized in $5 \%$ calcium hypochlorite for $15 \mathrm{~min}$ and washed with sterilized distilled water. Samples were germinated for 2 months in Petri dishes on a hormone-free 1/2 MS basic medium in the dark at 25 ${ }^{\circ} \mathrm{C}$. The percentage of protocorms was calculated in 3 repetitions of 100-200 seeds in each. The stages of development of protocorms were determined by the Vasudevan and van Staden (2010) method: 1. imbibed seeds, swollen, still covered by testa (= viable seeds) (Fig. 1D, E); 2. enlarged seeds without testa (=germination) (Fig. 1C); 3. protocorms with rhizoids (Fig. 1D); 4. protocorms with pointed shoot apex and rhizoids (appearance of shoot apex) (Fig. 1E). The stages of seed germination were recorded using a Keyence VHX-1000E light microscope.

The water content was determined by the weight loss of seeds (50 $\mathrm{mg}$ in 2 replicates) after drying to constant weight at $95^{\circ} \mathrm{C}$.

For cryogenic storage, the samples were partially dried in a sterile airflow at room temperature and 40$60 \%$ relative humidity for $2-6 \mathrm{~h}$ in the laminar box. The drying stopped when the sample weight was reduced by $60-70 \%$. The dehydrated seeds in sterile cryo-vials (Nunc, USA) were immersed in liquid nitrogen for 1 month.

The viability of seeds was also determined by vital staining with $0.005 \%$ fluorescein diacetate (FDA, Sigma-Aldrich, USA) or 1\% 2,3,5-triphenyltetrazolium chloride (TTC). The samples were stained on a glass slide in the dark wet chamber for 2 hours, washed several times with distilled water and mounted in $50 \%$ glycerol. Samples were investigated with Olympus FV1000D confocal microscope and Axioplan 2 light microscope. The fluorescence was excited with violet $(405 \mathrm{~nm})$ and blue $(473 \mathrm{~nm})$ lasers at $50 \%$ power. The signal was recorded in blue (425-460 nm), green (485-530 nm) and red (560-660 nm) channels.

D. nobile fruit is a dehiscent capsule (Fig. 1B). The time of seed ripening is 12-13 MAP.

The archesporial cell, the inner integument initials and megasporogenesis were noted at the age of 2 MAP. Maturation of the embryo sac and fertilization occurred at 2.5-3 MAP. A few-celled (2-4 cell) embryo formed at 3-3.5 MAP. The suspensor at this stage was still within the embryo sac (Fig. 2A). The developmental stages from zygote to multicellular embryo were found in fruits at 4-6 MAP. We observed the variability due to the uneven development of ovules, as well as the abortive ovules without an embryo (Fig. 2E).

At the fertilization stage (2-2.5 MAP), the ovule had two double integuments surrounding the embryo sac. The micropylar end turned towards the placenta (anatropic ovule). At the early embryogenesis stage (3 MAP), the inner part of the inner integument is covered with a cutinized layer (Fig. 2A). This layer had a reddish-brownish autofluorescence. It was black-brown when stained with FDA (Fig. 2B) and was almost invisible when stained with TTC (Fig. 2E). The cutinized shell was thin at the micropylar end and thicker at the chalazal end (Fig. 2A).

Seeds with suspensor inside and outside the embryo sac were identified on 4 MAPs (Fig. 2B, C). The first category included smaller multicellular embryos with vesicle suspensor. The second category included 
larger multicellular embryos with a branched unicellular suspensor. Living cells of the inner lining died at 5 MAP (Fig. 2C). Suspension desorption and the formation of a multicellular autonomous embryo were observed at 6 MAP (Fig. 2D). The protoplasts of the inner layer of the outer integument died at 7 MAP, and the formation of a full-fledged seed coat from the outer layer of the outer integument occurred at 8 MAP.

The table shows the parameters of seeds before immersion in liquid nitrogen and after cryogenic storage. The immature seeds at the initial stages of embryogenesis poorly retained moisture. Large water losses are associated with intensive morphogenetic processes, in particular, the suspensor formation and its exit outside the embryo sac. The immature seeds stabilized the ability to retain moisture after suspensor desorption.

During the germination of immature seeds at the stage of the first embryonic divisions (3-3.5 MAP), the embryos swelled and formed protocorms without rhizoids (Fig. 1C). No further development occurred.

Seeds at 4-5 MAP retained germination after drying (16\%) but lost germination after freezing. At 6 MAP, $30 \%$ of the seeds germinated in vitro after dehydration in airflow and only $10.7 \%$ of the seeds germinated after storage in liquid nitrogen. At 7 MAP, $81-94 \%$ of seeds acquired cryostability and retained their germination after cryogenic storage. Over $80 \%$ of seeds had stable germination both after drying and after cryogenic storage until full ripening and fruit opening at 12 MAP.

The earliest time that immature $D$. nobile seeds can be successfully stored in liquid nitrogen in a viable state and germinated if necessary is 6 MAP. A visual sign of seed maturation is the appearance of a yellow colour. The proportion of protocorm formation and further organogenesis is higher in seeds older than 6 MAP (Fig. 1E, F). However, due to many seeds in the fruit, their relatively low germination after cryogenic storage is not critical.

Fig. 2E, F shows the results of the TTC tests. The proportion of FDA or TTC-positive seeds and the staining dynamics did not correlate with the in vitro germination (Fig. 3, Table). The largest proportion of stained embryos was observed at 4-4.5 MAP. At this stage, the suspensor extends beyond the embryo sac. Vital dyes stained both less developed embryos with a rounded suspensor inside the embryo sac and embryos with a large branching suspensor outside it. Cutinization of the inner layer of the inner integument after fertilization probably not only fails to protect the embryo from excessive dehydration but is also permeable to vital dyes.

The proportion of coloured embryos decreased as the seeds matured. Staining decreases after suspensor desorption at 6 MAP. The cells of the inner integument died, their periclinal walls became denser, forming an impermeable membrane around the embryo. Thus, the multicellular embryo acquired autonomy, and the percentage of seed germination and cryostability increased.

The difficulties of seed reproduction of orchids in culture are associated with the morphological features of seeds (Rasmussen 1995). 
In D. nobile and its hybrids, the ability of seeds of different ages to germinate in vitro varies in different authors from 3-4 MAP (Vasudevan and van Staden 2010; Udomdee et al. 2014) to 6 MAP (PoddubnayaArnoldi 1964). In our work, immature seeds germinated in vitro at the age of 3 MAP. Early stages of development of the ovule were observed during this period, but mainly the formation of the embryo sac and fertilization.

Our study confirmed the ability of $D$. nobile seeds to germinate at 3 and 4 MAP, but the development stopped at the stage of protocorms without rhizoids.

Vital dye staining is often used to assess the viability of orchid seeds. The methods using TTC and FDA were described by Wood and Pritchard (2004) and Sawma and Mohler (2002) respectively. These tests are often used due to a simple assessment by counting coloured embryos. However, the time of seed staining, as well as the pre-soaking in distilled water or other substances (for example, when sterilized with $\left.\mathrm{Ca}(\mathrm{OCl})_{2}\right)$, significantly affect the intensity of staining and, therefore, distort the results of the tests (van Waes and Debergh 1986; Vujanovic et al. 2000). Our study showed that FDA and TTC staining did not correlate with the germination ability of $D$. nobile seeds. The increase in the number of FDA-stained embryos at the stage of active embryogenesis (4 MAP) in our work corresponds to the data of the authors who used TTC (Vasudevan and van Staden 2010).

Compared to most crop seeds, mature seeds of epiphytic orchids are short-lived. Today, the most longterm preservation of orchid seeds is possible in cryobanks at liquid nitrogen $\left(-196^{\circ} \mathrm{C}\right)$ (Engelmann 2004$)$.

Optimal seed moisture is crucial for viability. Several techniques are used to prepare immature seeds for freezing (Engelmann 2004; Hirano et al. 2005; Merritt et al. 2014). In our work, the seeds were dried in sterile airflow. In this case, the germination of seeds is influenced not only by freezing but also by dehydration (Makeen et al. 2005).

Thus, the fruits of $D$. nobile fully ripen and open at the age of 11-12 MAP, the seeds acquired resistance to freezing and drying in the airflow at the age of $6 \mathrm{MAP}$ and the optimal period for cryopreservation of immature seeds is 7 MAP, which reduces the time to achieve technical maturity by five months.

\section{Declarations}

Funding The study was carried out under Institutional research project №118021490111-5 at the Unique Scientific Installation "The Fund Greenhouse" of the Main Botanical Garden of the Russian Academy of Sciences and within the framework of a state assignment "Cryoresistance, cryopreservation and post cryogenic culture of plants" (state registration no. AAAA-A19-119042390111-0) with the partnership of Unique scientific installation: the Experimental Plant Cryobank at K.A. Timiryazev Institute of Plant Physiology of the Russian Academy of Sciences (Moscow, Russia)

Conflicts of interest/Completing The authors declare that they have no conflicts of interest. 
Availability of data and material All data transparency

Code availability Not applicable

Author's contributions The authors took an equal part in writing the manuscript.

\section{References}

1. Engelmann F (2004) Plant cryopreservation: progress and prospects. In Vitro Cellular \& Developmental Biology - Plant 40:427-433. https://doi.org/10.1079/IVP2004541

2. Hirano T, Godo T, Mii M, Ishikawa K (2004) Cryopreservation of immature seeds of Bletilla striata by vitrification. Plant Cell Reports 23:534-539. https://doi.org/10.1007/s00299-004-0893-9

3. Makeen MA, Noor NM, Dussert S, Clyde MM (2005). Cryopreservation of whole seeds and excised embryonic axes of Citrus suhuiensis cv. limau langkat in accordance to their desiccation sensitivity. CryoLetters 26(4):259-268.

4. Merritt DJ, Hay FR, Swarts ND, Sommerville KD, Dixon KW (2014) Ex situ conservation and cryopreservation of orchid germplasm. International Journal of Plant Sciences 175:46-58. https://doi.org/10.1086/673370

5. Poddubnaya-Arnoldi VA (1964) Comparatively-embryology investigation of representatives orchid family. Bull Main Bot Gard AS USSR 54:51-62.

6. Rasmussen HN (1995) Terrestrial Orchids: From Seed to Mycotrophic Plant. Cambridge University Press, Cambridge.

7. Sawma JT, Mohler CL (2002) Evaluating seed viability by an unimbibed seed crush test in comparison with the tetrazolium test. Weed Technology 16:781-786. https://doi.org/10.1614/0890037X(2002)016[0781:ESVBAU]2.0.CO;2

8. Udomdee W, Pei-Jung Wen P-J, Lee C-Y, Chin S-W, Chen F-C (2014) Effect of sucrose concentration and seed maturity on in vitro germination of Dendrobium nobile hybrids. Plant Growth Regul 72:249255. https://doi.org/10.1007/s10725-013-9856-x

9. van Waes JM, Debergh PC (1986) Adaptation of the tetrazolium method for testing the seed viability, and scanning electron microscopy study of some western European orchids. Physiol Plant 66:435442. https://doi.org/10.1111/j.1399-3054.1986.tb05947.x

10. Vasudevan R, van Staden JV (2010) Fruit harvesting time and corresponding morphological changes of seed integuments influence in vitro seed germination of Dendrobium nobile Lindl. Plant Growth Regul 60:237-246. https://doi.org/10.1007/s10725-009-9437-1

11. Vujanovic V, St-Arnaud M, Barabe D, Thibeault G (2000) Testing of Orchid Seed and the Promotion of Coloration and Germination. Annals of Botany 86:79-86. https://doi.org/10.1006/anbo.2000.1162

12. Wood CB, Pritchard HW (2004) Determination of intra-specific variation in orchid seed viability using fluorescein diacetate. Seed Science and Technology 32:629635.https://doi.org/10.15258/sst.2004.32.2.32 


\section{Tables}

Table. Germination of $D$. nobile seeds of different ages after drying in airflow, cryogenic storage and two months of in vitro culture

\begin{tabular}{|llllll|}
\hline $\begin{array}{l}\text { Age, } \\
\text { MAP }\end{array}$ & $\begin{array}{l}\text { Initial } \\
\text { humidity, } \\
\%\end{array}$ & $\begin{array}{l}\text { Humidity after } \\
\text { drying, } \%\end{array}$ & $\begin{array}{l}\text { Initial } \\
\text { germination*, } \\
\%\end{array}$ & $\begin{array}{l}\text { Germination } \\
\text { after drying, } \\
\%\end{array}$ & $\begin{array}{l}\text { Germination after } \\
\text { cryogenic storage, } \%\end{array}$ \\
\hline 2 & 92,3 & 75,5 & 0 & 0 & 0 \\
\hline 3 & 89,2 & 63,2 & $21,7 \pm 6^{*}$ & 0 & 0 \\
\hline 4 & 85,4 & 11,7 & $19,0 \pm 1^{*}$ & $16,0 \pm 3^{\star}$ & 0 \\
\hline 5 & 86,0 & 13,1 & $26,3 \pm 1^{\star \star}$ & $\begin{array}{l}\text { single } \\
\text { protocorms }\end{array}$ & single protocorms** \\
\hline 6 & 80,1 & 19,0 & $33,7 \pm 4$ & $30,3 \pm 1$ & $10,7 \pm 2$ \\
\hline 7 & 70,9 & 17,8 & $82,3 \pm 2$ & 82 & $81,3 \pm 1$ \\
\hline 8 & 52,3 & 29,2 & $98,3 \pm 1$ & $90 \pm 1$ & $87,7 \pm 1$ \\
\hline 10 & 28,1 & 24,5 & $94,7 \pm 4$ & $90 \pm 2$ & $93,7 \pm 2$ \\
\hline 12 & 43,3 & 21,1 & $94,3 \pm 2$ & $89 \pm 2$ & $89,7 \pm 2$ \\
\hline
\end{tabular}

The most developed protocorms in the sample: * - protocorms without rhizoids; ** - protocorms with rhizoids; without asterisks - protocorms with rhizoids and apical bud

\section{Figures}



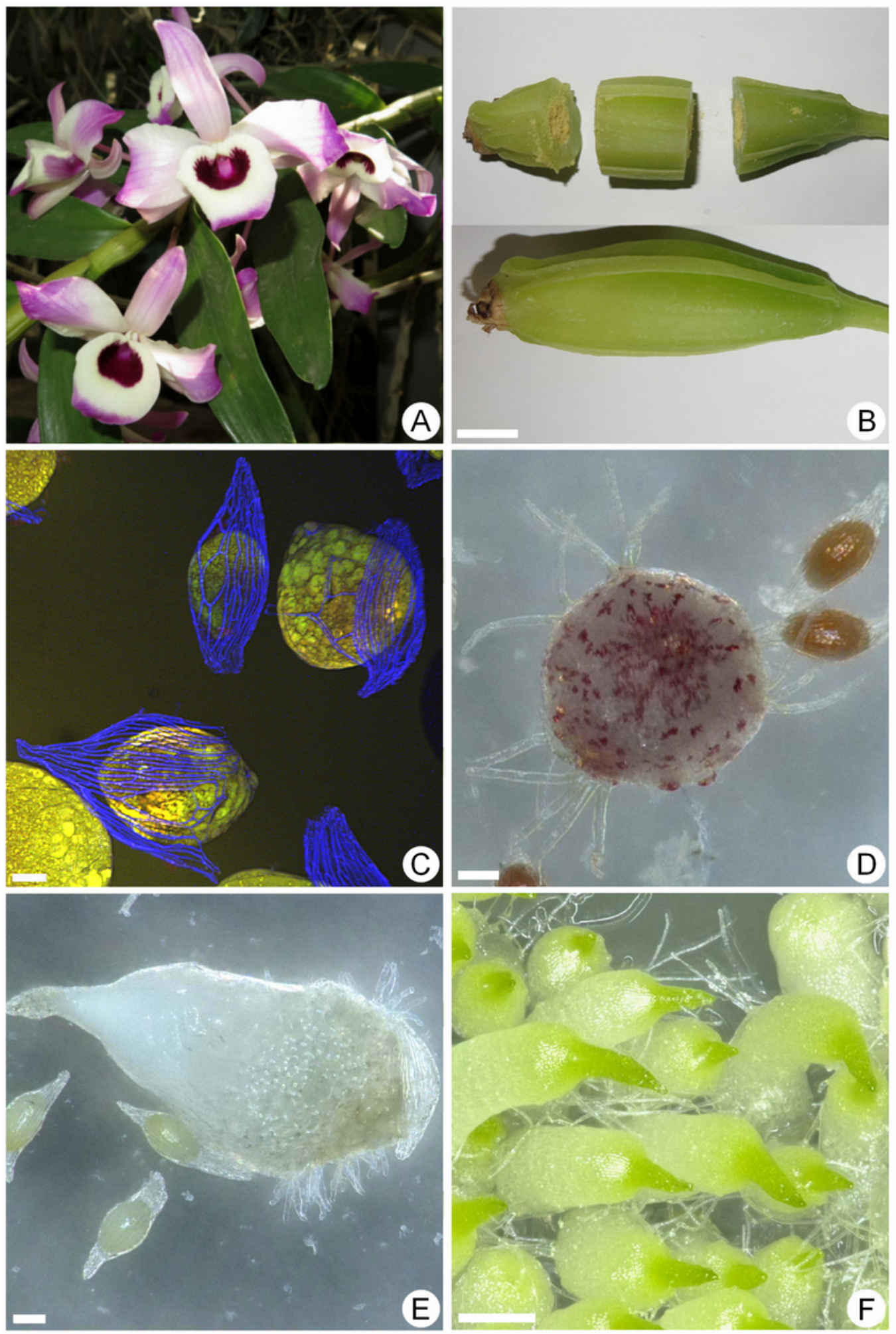

\section{Figure 1}

Flowers, fruits and protocorms of D. nobile. A Flowers; B mature fruits at 12 MAP; C enlarged protocorms without rhizoids (FDA, confocal microscopy); D protocorm with rhizoids; E protocorm with pointed shoot apex and rhizoids (cultivation in the dark 4 weeks); $F$ protocorms with pointed shoot apex and rhizoids, germinated 3 weeks in the dark and 1 week in the light. D-F Keyence VHX-1000E light microscopy. Scale bars: $B=1000 \mu \mathrm{m} ; C-E=100 \mu \mathrm{m} ; F=500 \mu \mathrm{m}$. 


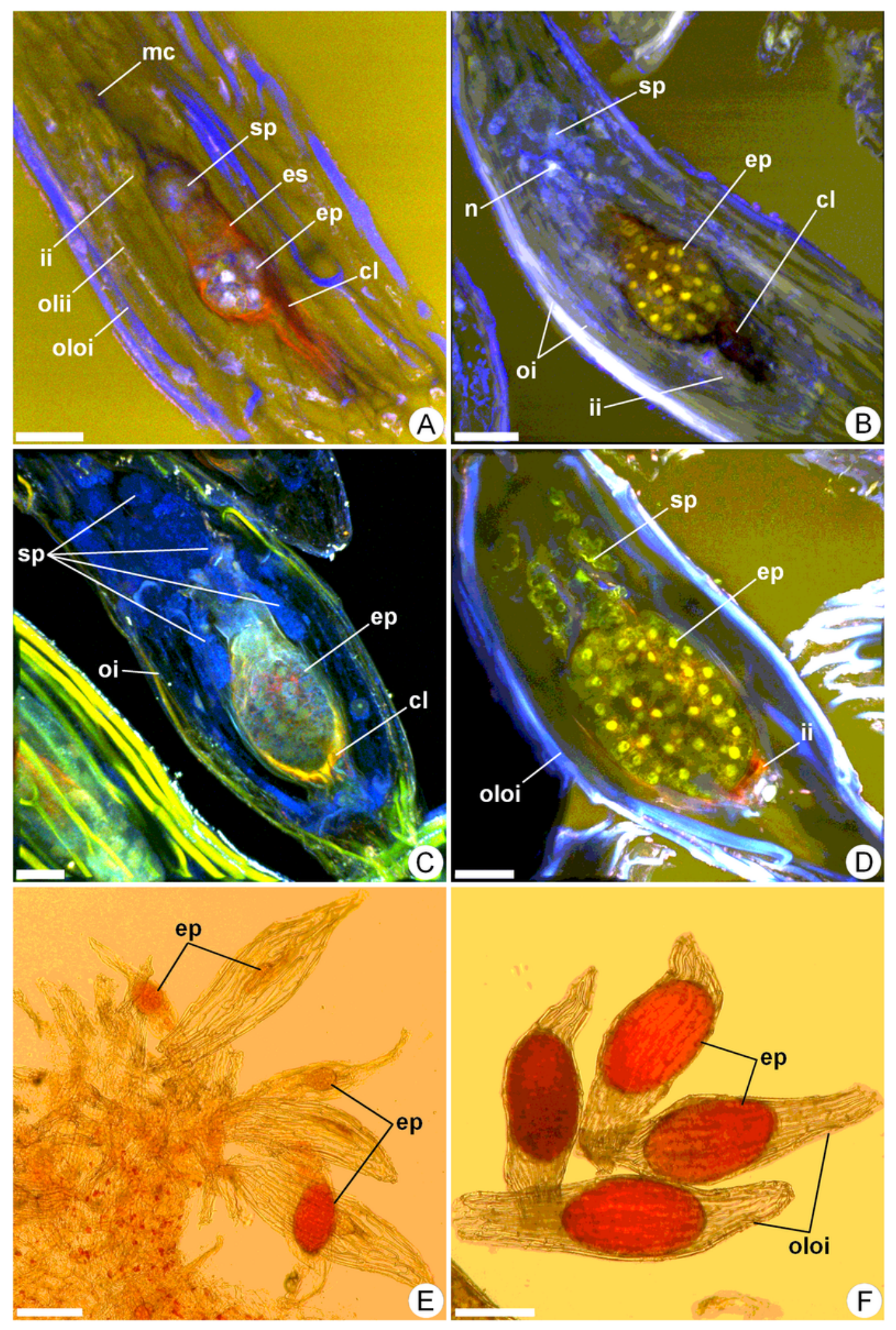

\section{Figure 2}

Ovules and seeds of D. nobile. Confocal microscopy: A the suspensor of the few-celled embryo does not extend the embryo sac, the cutinized inner layer of the inner integument fluoresces red-brown (3 MAP) (FDA); B the suspensor extends beyond the embryo sac, the nuclei of the embryo proper fluoresce yellow, the nucleus of the suspensor fluoresces blue, the dark cutinized shell of the inner layer of the inner integument does not fluoresce (4 MAP) (FDA); C the branched suspensor fluoresces blue after elimination 
of the inner integument (5 MAP) (Auto); D elimination of the inner layer of the outer integument and the suspensor, nuclei of the embryo proper fluoresce yellow (6 MAP, FDA). Light microscopy (Axioplan 2): E and $F$ the seeds of D. nobile, stained with TTC at 5 MAP (E) and 8 MAP (F). Scale bars: $A=20 \mu \mathrm{m} ; B-F=$ $100 \mu \mathrm{m} ; \mathrm{D}=30 \mu \mathrm{m}$. cl, cutinized layer; ep, embryo proper; es, embryo sac; ii, inner integument; mc, micropyle; n, nucleus; oi, outer integument; olii, outer layer of inner integument; oloi, outer layer of outer integument; sp, suspensor

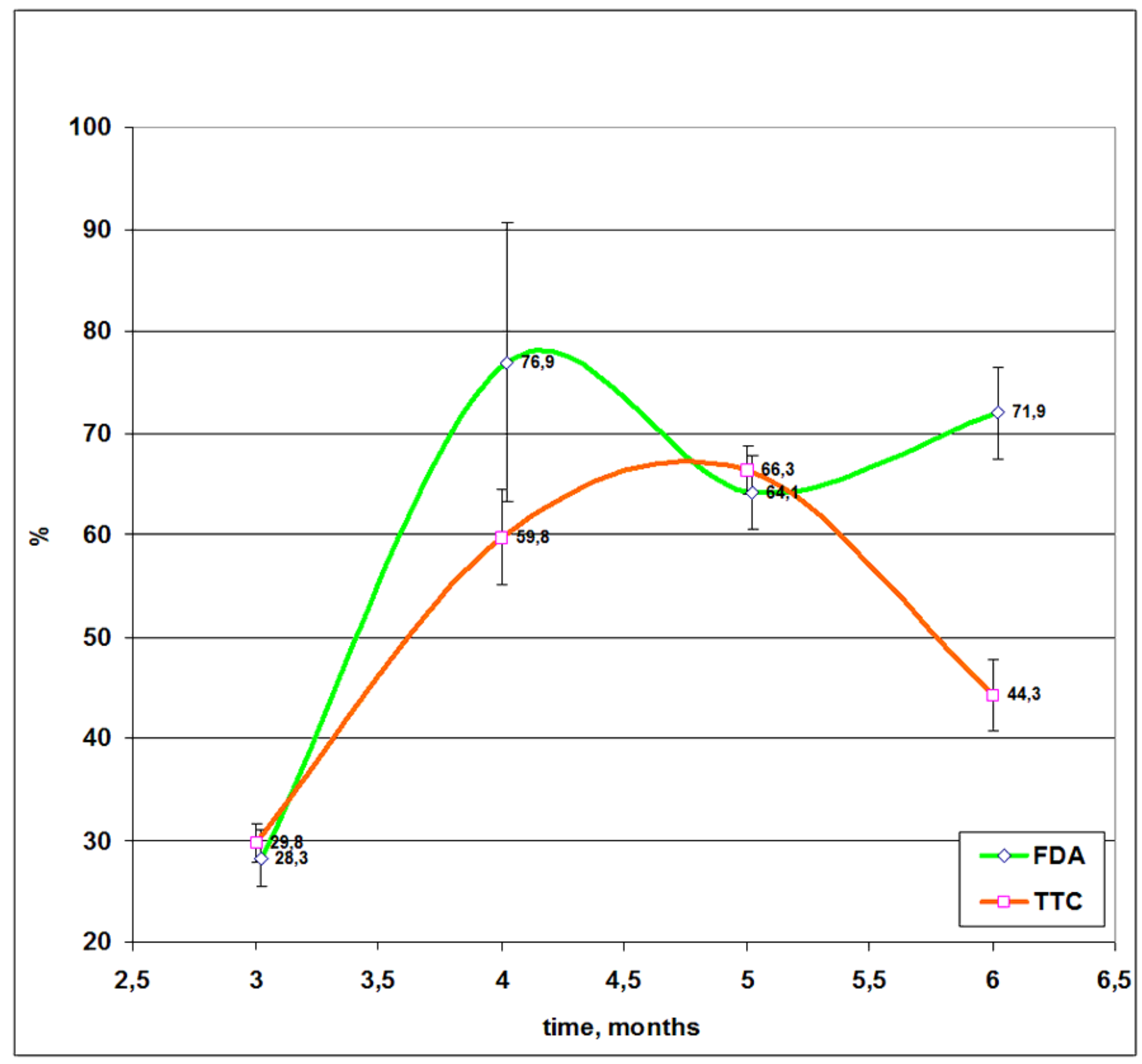

\section{Figure 3}

Embryo staining with FDA and TTC vital dyes as a percentage of the number of seeds and ovules with an embryo at the age of 3-6 MAP 